\title{
REPRESENTATIONS OF HECKE ALGEBRAS
}

\author{
EUGENE GUTKIN
}

\begin{abstract}
We find all operators of a certain type that satisfy the braid relations corresponding to any generalized Cartan matrix.
\end{abstract}

1. Introduction. Let $\mathfrak{h}$ be a simple complex Lie algebra, let $\mathfrak{h}$ be a Cartan subalgebra, let $W$ be the Weyl group generated by the simple reflections $s_{i}$ and let $\alpha_{i}$ be the simple roots, $i=1, \ldots$, rk g. Bernstein, Gelfand and Gelfand in [3] and, independently, Demazure in [5] introduced the operators

$$
B_{i}=\left(1-s_{i}\right) / \alpha_{i}
$$

on the space of polynomials on $\mathfrak{h}$. In [6] Demazure wrote another set of operators

$$
D_{i}=\left(1-s_{i}\right) /\left(e^{\alpha_{i}}-1\right)
$$

which act on the space of meromorphic functions on $\mathfrak{h}$. These operators have the following remarkable property. If $w \in W$ and $w=s_{i_{1}} \cdots s_{i_{n}}$ is a reduced decomposition then the operators $B_{w}=B_{i_{1}} \cdots B_{i_{n}}$ and $D_{w}=D_{i_{1}} \cdots D_{i_{n}}$ depend only on $w$ and not on the choice of the decomposition of $w$. The defining relations of $W$ are $s_{i}^{2}=1$ and $\left(s_{i} s_{j}\right)^{m_{i j}}=1$ for $i \neq j$ where $m_{i j}$ is the order of $s_{i} s_{j}$ and $m_{i j}=0$ if $s_{i} s_{j}$ has an infinite order. In particular we have the relations (with $m_{i j}$ factors on each side)

$$
s_{i} s_{j} s_{i} \cdots=s_{j} s_{i} s_{j} \cdots
$$

which are called the braid relations. The property of $B_{i}$ and $D_{i}$ that we mentioned above is equivalent, by [4], to the fact that they satisfy the braid relations

$$
B_{i} B_{j} B_{i} \cdots=B_{j} B_{i} B_{j} \cdots, \quad D_{i} D_{j} D_{i} \cdots=D_{j} D_{i} D_{j} \cdots .
$$

The BGG operators $B_{i}$ and the Demazure operators $D_{i}$ have a geometric nature. They appeared (respectively) in the study of the cohomology ring and the $K$-ring of the flag manifold associated with $\mathfrak{g}$. Since [3, 5 and 6], operators satisfying braid relations arose in many other contexts. First of all, the BGG operators were generalized to the Kac-Moody algebras and used in the study of their (infinite dimensional) flag varieties (see $[1,10,11,13,14])$. Operators satisfying the braid relations also appeared in connection with the Bethe Ansatz for certain quantum Hamiltonians associated with Weyl groups [8]. These operators are closely related to the BGG operators [9], more precisely, they are adjoint to the operators

$$
G_{i}=t B_{i}+s_{i}=t / \alpha_{i}-\left(-1+t / \alpha_{i}\right) s_{i}
$$

Received by the editors June 17, 1987.

1980 Mathematics Subject Classification (1985 Revision). Primary 22E30; Secondary 16A64.

Partially supported by NSF Grant DMS-8600350. 
where $t$ is an arbitrary complex number. Then Lusztig in his work on representation theory [15] introduced the operators ( $q$ is a parameter)

$$
L_{i}=(1-q) D_{i}+q s_{i}=\frac{1-q}{e^{\alpha_{i}}-1}-\left(-q+\frac{1-q}{e^{\alpha_{i}}-1}\right) s_{i}
$$

and showed that they satisfy the braid relations.

The reader probably noticed that all these operators have the form

$$
T_{i}=f\left(\alpha_{i}\right)+g\left(\alpha_{i}\right) s_{i}
$$

where $f$ and $g$ are certain meromorphic functions. In view of these examples, it is natural to ask for which functions $f$ and $g$ operators (7) satisfy the braid relations of an arbitrary root system. Recently, Bressler and Evens [7] found the answer under the assumption that in the root system there are two simple roots with the $120^{\circ}$ angle between them. In the present work we extend their result to the arbitrary root systems. The answer, roughly speaking, is that the examples above exhaust all the possibilities.

The argument of our main theorem is self-contained, hence, it provides an alternative proof of the results of $[\mathbf{3}, \mathbf{5}, \mathbf{6}, \mathbf{7}, \mathbf{9}$ and 15] mentioned above. $\S 2$ contains the main result and in $\S 3$ we give a few applications of it. One of them answers a question of J. Bernstein [2].

2. Main result. We start by establishing the necessary notation. Let $A$ be a generalized $n \times n$ Cartan matrix, denote by $W$ the corresponding Weyl group with the set $s_{1}, \ldots, s_{n}$ of simple reflections and let $\alpha_{1}, \ldots, \alpha_{n}$ be the simple roots (cf. [12]). For any pair $i \neq j$ set $m_{i j}$ equal to $2,3,4$ or 6 if the product $a_{i j} a_{j i}$ is equal to $0,1,2$ or 3 respectively. If $a_{i j} a_{j i} \geq 4$ set $m_{i j}=0$. It is well known (see, e.g., [12]) that $m_{i j}>0$ is the order of $s_{i} s_{j}$ and $m_{i j}=0$ if and only if $s_{i} s_{j}$ has an infinite order.

The braid algebra $B$ is generated by $b_{1}, \ldots, b_{n}$ with the defining relations

$$
b_{i} b_{j} b_{i} \cdots=b_{j} b_{i} b_{j} \cdots
$$

with $m_{i j}$ factors on each side for every $i<j$. We say that $B$ is "nontrivial" if $m_{i j}>2$ for some pair $i \neq j$.

Let $\mathfrak{h}$ be a linear space, $\alpha$ a linear form on $\mathfrak{h}$ and $f$ a meromorphic function of one complex variable. Notation $f(\alpha)$ means the function on $\mathfrak{h}$ given by $f(\langle\alpha, x\rangle), x \in$ $\mathfrak{h}$. We denote by $M$ the algebra generated by all such functions and call $M$ the algebra of meromorphic functions on $\mathfrak{h}$. In what follows $\mathfrak{h}$ will be the Cartan algebra $(\operatorname{dim} \mathfrak{h}=n$ ) corresponding to $A$. The group $W$ naturally acts by automorphisms of $M$. Denote by $M_{W}$ the smash product of $M$ and $W[14]$. The algebra $M_{W}$ is a free module over $M$ with the basis $w, w \in W$. If $f \rightarrow w \cdot f$ denotes the action of $W$ on $M$ then the defining relations of $M_{w}$ are $w f w^{-1}=w \cdot f$. We call elements of $M_{W}$ operators because $M_{W}$ is naturally isomorphic to the algebra of linear operators on $M$ generated by the multiplication operators $f$ and by the operators $w \in W$.

DEFINITION 1. Let $f$ and $g$ be meromorphic functions of one variable. We say that the pair $(f, g)$ represents the braid relations (corresponding to $A$ ) if the operators

$$
T_{i}=f\left(\alpha_{i}\right)+g\left(\alpha_{i}\right) s_{i}, \quad i=1, \ldots, n,
$$

in $M_{W}$ satisfy the braid relations (8). The representation is called nontrivial if $f, g \neq 0$. 
THEOREM 1. Let $A$ be a generalized Cartan matrix with a nontrivial braid algebra $B$. A pair $(f, g)$ gives a nontrivial representation of the braid relations if and only if one of the following two cases holds.

$$
\begin{aligned}
& \text { Case (i) } f(x)=b / x, \quad g(x)=h(x)(c+b / x) . \\
& \text { Case (ii) } f(x)=b /\left(e^{a x}-1\right), \quad g(x)=h(x)\left(c+b /\left(e^{a x}-1\right)\right) .
\end{aligned}
$$

In both cases $a \neq 0, b$ and $c$ are arbitrary constants and $h$ is any meromorphic function satisfying

$$
h(x) h(-x)=1 .
$$

In what follows we call the two cases above the BGG case and the Demazure case respectively.

PROOF. The braid relation

$$
T_{i} T_{j} T_{i} \cdots=T_{j} T_{i} T_{j} \cdots
$$

corresponding to a pair $i \neq j$ involves only the subsystem of the root system generated by $\alpha_{i}$ and $\alpha_{j}$ and the corresponding subgroup of the Weyl group. If $m_{i j}=0$ there is no relation and any operator of the form (9) satisfies (13) for $m_{i j}=2$. Therefore we can assume that our root system has rank 2 , denote $m_{i j}$ by $m$ and consider the three possibilities: $A_{2}(m=3)$ or $B_{2}(m=4)$ or $G_{2}(m=6)$ in the notation of [3]. We denote the simple roots by $\alpha$ and $\beta$ where $\beta$ is the longer one (if $m=4$ or 6 ). We set

$$
T_{\alpha}=f(\alpha)+g(\alpha) s_{\alpha}, \quad T_{\beta}=f(\beta)+g(\beta) s_{\beta} .
$$

It remains to show that in each of the three cases the equation

$$
T_{\alpha} T_{\beta} T_{\alpha} \cdots=T_{\beta} T_{\alpha} T_{\beta} \cdots
$$

with $m$ factors is satisfied if and only if the pair $(f, g)$ is given by (i) or (ii) of the theorem.

Equation (15) is equivalent to a system of functional equations on $f$ and $g$. We begin by observing the features of this system common to all three cases. Substituting (14) into (15) and multiplying out we obtain $2^{m}$ monomials in each side of (15). We label these monomials by the subsets $X \subset\{1, \ldots, m\}$ where we agree to take the $g s$ factor on $i$ th place if $i \in X$ and take the $f$ factor otherwise.

Denote by $l(w)$ the length function on $W$ and by $w_{0}$ the unique longest element, $l\left(w_{0}\right)=m$ which has two expressions

$$
w_{0}=s_{\alpha} s_{\beta} s_{\alpha} \cdots=s_{\beta} s_{\alpha} s_{\beta} \cdots .
$$

Denote by $w(L, X)$ and $w(R, X)$ the Weyl group elements obtained from $w_{0}$ by deleting from the left-hand side (LHS) and the right-hand side (RHS) of (16) the $i$ th factors for $i \notin X$. Commute the $s_{\alpha}, s_{\beta}$ factors in (15) to the right using the relations of $M_{W}$. Then the monomial labelled by $X$ in the LHS of (15) becomes $g\left(\gamma_{1}\right) \cdots g\left(\gamma_{k}\right) f\left(\gamma_{k+1}\right) \cdots f\left(\gamma_{m}\right) w(L, X)$ and in RHS we get

$$
g\left(\delta_{1}\right) \cdots g\left(\delta_{k}\right) f\left(\delta_{k+1}\right) \cdots f\left(\delta_{m}\right) w(R, X)
$$

where $\gamma_{1}, \ldots, \gamma_{m}, \delta_{1}, \ldots, \delta_{m}$ are some roots and $k=|X|$. 
Collecting together the terms in (15) with $w(L, X)=w(R, Y)=w$ and using that there are no relations between $w$ 's we obtain that (15) is equivalent to $2 m=$ $|W|$ functional equations $E_{w}$ on $f$ and $g$. Each equation $E_{w}$ is homogeneous of degree $m$ in $f$ and $g$.

Apart from $l(1)=0$ and $l\left(w_{0}\right)=m$ for every $1 \leq l \leq m-1$ there are two elements in $W$ of length $l$ and the involution $\bar{s}_{\alpha}=s_{\beta}$ interchanges them. Equations $E_{w}$ and $E_{\bar{w}}$ are equivalent which leaves us with the system of $m+1$ equations $E_{w}$, one for each value of $l=l(w), 0 \leq l \leq m$. To avoid confusing $w$ with $\bar{w}$ we consider the elements $w$ which start with $s_{\alpha}: w=1, s_{\alpha}, s_{\alpha} s_{\beta}, \ldots$

With a reduced word $w=s_{\alpha} s_{\beta} s_{\alpha} \cdots$ of length $l$ one associates the sequence $\gamma_{1}=\alpha, \gamma_{2}=s_{\alpha} \beta, \gamma_{3}=s_{\alpha} s_{\beta} \alpha, \ldots, \gamma_{l}$ of roots. It is well known (see e.g. [4]) that the set $\left\{\gamma_{1}, \ldots, \gamma_{l}\right\}=\Delta_{+} \cap w \Delta_{-}$, thus it depends only on $w$. Our analysis of (15) shows that every monomial in $E_{w}$ contains the factor $\prod_{i=1}^{l(w)} g\left(\gamma_{i}\right)$ where $\gamma_{i}$ runs through $\Delta_{+} \cap w \Delta_{-}$. Since $g \neq 0$ we divide $E_{w}$ by this common factor and denote the obtained equation by $E_{w}$ again. The new $E_{w}$ is homogeneous in $f$ and $g$ of degree $m-l(w)$. The equations of degree zero and one are trivial. They are $1=1$ and $f(\beta)=f(\beta)$ respectively. This leaves us with a system of $m-1$ equations of degrees $2,3, \ldots, m$ which we denote from now on by $E_{2}, \ldots, E_{m}$.

The $g$-factors come into our equations in pairs $g(\gamma) g(-\gamma)$ due to the phenomenon of collapsing in the words of $W$. For instance, let $m=4$ and consider the monomial in the LHS of (15) obtained by deleting the second $s_{\alpha}$ in $s_{\alpha} s_{\beta} s_{\alpha} s_{\beta}$. We have

$$
\begin{aligned}
g(\alpha) s_{\alpha} g(\beta) s_{\beta} f(\alpha) g(\beta) s_{\beta} & =g(\alpha) s_{\alpha} f\left(s_{\beta} \alpha\right) g(\beta) g(-\beta) s_{\beta}^{2} \\
& =g(\alpha) f\left(s_{\alpha} s_{\beta} \alpha\right) g\left(s_{\alpha} \beta\right) g\left(-s_{\alpha} \beta\right) s_{\alpha}
\end{aligned}
$$

and after dividing $g(\alpha) s_{\alpha}$ out we obtain the term $f\left(s_{\alpha} s_{\beta} \alpha\right) g\left(s_{\alpha} \beta\right) g\left(-s_{\alpha} \beta\right)$ in $E_{3}$. Thus, the $g$-part of this monomial has the predicted form with $\gamma=s_{\alpha} \beta$. It is clear from the preceding example that each time we get a factor $g(\gamma) g(-\gamma)$ we also get an $f$-factor. Therefore, $E_{2}$ has no $g$-factors, it is a quadratic equation in $f$.

At this point we turn to the case by case analysis of the three systems of functional equations starting with the most difficult case $m=6$. Reflection about the midpoint interchanges the words $s_{\alpha} s_{\beta} s_{\alpha} s_{\beta} s_{\alpha} s_{\beta}$ and $s_{\beta} s_{\alpha} s_{\beta} s_{\alpha} s_{\beta} s_{\alpha}$. As a consequence of this, equation $E_{6}$ is trivial which leaves us with $E_{2}, \ldots, E_{5}$ written below. They are

$$
\begin{aligned}
& f(\alpha) f(\beta)+f(3 \alpha+\beta) f(-\alpha)+f(2 \alpha+\beta) f(-3 \alpha-\beta) \\
& +f(3 \alpha+2 \beta) f(-2 \alpha-\beta)+f(\alpha+\beta) f(-3 \alpha-2 \beta) \\
& =f(\beta) f(\alpha+\beta) \text {, } \\
& f^{2}(3 \alpha+2 \beta) f(-2 \alpha-\beta)+f(2 \alpha+\beta) f(-3 \alpha-\beta) f(3 \alpha+2 \beta) \\
& +f(\alpha) f(\beta) f(3 \alpha+2 \beta) \\
& +f(3 \alpha+\beta) f(-\alpha) f(3 \alpha+2 \beta)+f(\alpha+\beta) g(3 \alpha+2 \beta) g(-3 \alpha-2 \beta) \\
& =f(\beta) f(3 \alpha+2 \beta) f(-2 \alpha-\beta)+f(\beta) f(2 \alpha+\beta) f(-3 \alpha-\beta) \\
& +f(\beta) f(3 \alpha+\beta) f(-\alpha)+f^{2}(\beta) f(\alpha)+f(\alpha+\beta) g(\beta) g(-\beta),
\end{aligned}
$$


(19)

$$
\begin{aligned}
f^{2}(2 \alpha+ & \beta) f^{2}(-3 \alpha-\beta)+f(\alpha) f(\beta) f(2 \alpha+\beta) f(-3 \alpha-\beta) \\
& +f^{2}(\alpha) f^{2}(\beta)+f^{2}(3 \alpha+\beta) f^{2}(-\alpha)+f(\alpha) f(-\alpha) f(\beta) f(3 \alpha+\beta) \\
& +f(3 \alpha+\beta) f(-3 \alpha-\beta) f(2 \alpha+\beta) f(-\alpha) \\
& +f(2 \alpha+\beta) f(-\alpha) g(3 \alpha+\beta) g(-3 \alpha-\beta) \\
& +f(3 \alpha+2 \beta) f(-3 \alpha-\beta) g(2 \alpha+\beta) g(-2 \alpha-\beta) \\
& +f(3 \alpha+\beta) f(\beta) g(\alpha) g(-\alpha)+f(\alpha) f(\alpha+\beta) g(\beta) g(-\beta) \\
= & f(\beta) f^{2}(2 \alpha+\beta) f(-3 \alpha-\beta)+f(\alpha) f^{2}(\beta) f(2 \alpha+\beta) \\
& +f(\beta) f(2 \alpha+\beta) f(3 \alpha+\beta) f(-\beta)+f(\beta) f(3 \alpha+\beta) g(2 \alpha+\beta) g(-2 \alpha-\beta) \\
& +f(\alpha+\beta) f(2 \alpha+\beta) g(\beta) g(-\beta)
\end{aligned}
$$

and

(20)

$$
\begin{aligned}
f^{2}(- & \alpha) f^{3}(3 \alpha+\beta)+f^{2}(3 \alpha+\beta) f(\beta) g(\alpha) g(-\alpha) \\
& +f^{2}(2 \alpha+\beta) f(-3 \alpha-\beta) g(3 \alpha+\beta) g(-3 \alpha-\beta) \\
& +f(3 \alpha+2 \beta) g(2 \alpha+\beta) g(-2 \alpha-\beta) g(3 \alpha+\beta) g(-3 \alpha-\beta) \\
& +f(\alpha) f(-\alpha) f(\beta) f^{2}(3 \alpha+\beta)+f(\alpha) f(\beta) f(2 \alpha+\beta) g(3 \alpha+\beta) g(-3 \alpha-\beta) \\
& +f^{2}(\alpha) f^{2}(\beta) f(3 \alpha+\beta)+f(\alpha) f(\alpha+\beta) f(3 \alpha+\beta) g(\beta) g(-\beta) \\
& +2 f(2 \alpha+\beta) f(-\alpha) f(3 \alpha+\beta) g(3 \alpha+\beta) g(-3 \alpha-\beta) \\
= & f^{2}(\alpha) f^{3}(\beta)+f^{2}(\alpha+\beta) f(-\beta) g(\beta) g(-\beta) \\
& +f^{2}(\beta) f(3 \alpha+\beta) g(\alpha) g(-\alpha)+2 f(\alpha) f(\beta) f(\alpha+\beta) g(\beta) g(-\beta) \\
& +f(3 \alpha+2 \beta) g(\alpha+\beta) g(-\alpha-\beta) g(\beta) g(-\beta) \\
& +f(\alpha) f^{2}(\beta) f(3 \alpha+\beta) f(-\alpha)+f(\beta) f^{2}(-\alpha) f^{2}(3 \alpha+\beta) \\
& +f(\alpha+\beta) f(3 \alpha+\beta) f(-\alpha) g(\beta) g(-\beta) \\
& +f(\beta) f(2 \alpha+\beta) f(-\alpha) g(3 \alpha+\beta) g(-3 \alpha-\beta) .
\end{aligned}
$$

Equation (17) is crucial. Assuming that $f$ is regular at zero and $f \neq 0$ we write $f(x)=a x^{n}+O\left(x^{n+1}\right), a \neq 0$. Substituting into (17) we get

$$
P_{2 n}(\alpha, \beta)+o\left(\alpha^{n}, \beta^{n}\right)=0
$$

where $P_{2 n}$ is a nonzero polynomial of degree $2 n$ in $\alpha$ and $\beta$. This contradiction shows that either $f=0$ or $f$ has a pole at zero. We consider the case $f \neq 0$ and we set $f=F^{-1}$ where $F(0)=0$. Setting $\alpha+\beta=x, \alpha=y$ we obtain from (17) after elementary transformations

$$
\begin{aligned}
\frac{F(y)-F(x)}{F(x-y)}= & \frac{F(x) F(y)}{F(x+2 y) F(-y)}+\frac{F(y)}{F(-2 x-y)} \\
& +\frac{F(x) F(y)}{F(x+y) F(-x-2 y)}+\frac{F(x) F(y)}{F(2 x+y) F(-x-y)} .
\end{aligned}
$$

Differentiating (21) with respect to $x$ and setting $x=0$ we obtain

$$
-F^{\prime}(0)\left[\frac{2}{F(-y)}+\frac{F(y)}{F(-y) F(2 y)}+\frac{1}{F(-2 y)}\right]=\frac{3 F^{\prime}(-y) F(y)}{F^{2}(-y)} \text {. }
$$


If $F^{\prime}(0)=0$, then, by $(22), F^{\prime}=0$, i.e. $F$ is constant. Since $F(0)=0, F=0$ which is a contradiction. Thus, $F^{\prime}(0) \neq 0$ and, normalizing, we can assume that $F^{\prime}(0)=1$. Equation (22) simplifies to

$$
\begin{gathered}
3 F^{\prime}(x) F(-x) F(2 x) F(-2 x)+2 F(x) F(2 x) F(-2 x) \\
+F(x) F(-x) F(2 x)-F^{2}(x) F(-2 x)=0 .
\end{gathered}
$$

Setting $F(x)=x G(x)$ we replace (23) with

$$
\begin{aligned}
6 x G^{\prime}(x) G(-x) G(2 x) G(-2 x)+6 G(x) G(-x) G(2 x) G(-2 x) \\
-4 G(x) G(2 x) G(-2 x)-G(x) G(-x) G(2 x)+G^{2}(x) G(-2 x)=0
\end{aligned}
$$

and the condition $G(0)=1$. We look for solutions of $(24)$ in the form

$$
G(x)=\sum_{n=0}^{\infty} a_{n} x^{n}, \quad a_{0}=1 .
$$

Substituting (25) into (24) we obtain the following sequence of equations on $a_{n}$.

$$
\left[2^{n}\left(1+(-1)^{n} 3\right)+3+(-1)^{n} 5+6 n\right] a_{n}=\varphi_{n}\left(a_{1}, \ldots, a_{n-1}\right)
$$

where $\varphi_{n}$ is a polynomial without constant term and $\varphi_{1}=0$. For $n=1,(26)$ becomes $0 \cdot a_{1}=0$, i.e. $a_{1}$ is arbitrary. For even $n$ the coefficient in (26) is positive, for odd $n$ it is equal to $-2\left(2^{n}+1-3 n\right)$. It is elementary to check that $n=1$ is the only integer solution of $2^{n}+1-3 n=0$. Hence, a formal power series solution of (24) is completely determined by $a_{1}$ and $G(x)=1$ if $a_{1}=0$. Therefore, a solution $F$ of (23), if it exists, is determined by $F^{\prime \prime}(0)=a$ and $F(x)=x$ if $a=0$. It is straightforward to check that $F(x)=x$ and for $a \neq 0$ the functions $F(x)=a^{-1}\left(e^{a x}-1\right)$ satisfy (23), hence they are the only solutions of (23). Checking by substitution that $f(x)=b / x$ and $f(x)=b\left(e^{a x}-1\right)^{-1}$ satisfy (17) we conclude that they are the only solutions.

To solve (18) we observe that 8 out of 10 terms of (18) do not contain the unknown function $g$. Switching these 8 terms to the LHS of the equation and setting $f$ to be $b x^{-1}$ or $b\left(e^{a x}-1\right)^{-1}$ we obtain by a straightforward computation the identity

$$
\text { LHS }=f(\alpha+\beta)[f(\beta) f(-\beta)-f(3 \alpha+2 \beta) f(-3 \alpha-2 \beta)] .
$$

Therefore, (18) becomes

(28) $g(\beta) g(-\beta)-g(3 \alpha+2 \beta) g(-3 \alpha-2 \beta)=f(\beta) f(-\beta)-f(3 \alpha+2 \beta) f(-3 \alpha-2 \beta)$

where $f$ is as above. Equation (28) is equivalent to

$$
g(x) g(-x)=f(x) f(-x)+d
$$

where $d$ is an arbitrary constant and using that

$$
f(x)+f(-x)=0
$$

in the BGG case and

$$
f(x)+f(-x)=-b
$$

in the Demazure case we rewrite (29) as

$$
g(x) g(-x)=(c+f(x))(c+f(-x))
$$


where $c^{2}=d$ in the BGG case, $c^{2}-b c=d$ in the Demazure case. By (32), the ratio $h(x)=g(x)[c+f(x)]^{-1}$ satisfies

$$
h(x) h(-x)=1 \text {. }
$$

The argument above can be obviously reversed which shows that the general solution $(f, g)$ of $(17)$ and (18) (provided $f, g \neq 0)$ is given by (i) and (ii) of the theorem. Substituting (i) and (ii) into (19) and (20) we obtain that they are also satisfied. This finishes the $m=6$ case.

In the case $m=4$ the equation $E_{4}$ is trivial just like in the previous case and the remaining equations $E_{2}$ and $E_{3}$ are

$$
f(\alpha) f(\beta)+f(a+\beta) f(-2 \alpha-\beta)+f(2 \alpha+\beta) f(-\beta)=f(\beta) f(\alpha+\beta)
$$

and

$$
\begin{gathered}
f^{2}(2 \alpha+\beta) f(-\alpha)+f(\alpha) f(\beta) f(2 \alpha+\beta)+f(\alpha+\beta) g(2 \alpha+\beta) g(-2 \alpha-\beta) \\
=f^{2}(\beta) f(\alpha)+f(\beta) f(2 \alpha+\beta) f(-\alpha)+f(\alpha+\beta) g(\beta) g(-\beta) .
\end{gathered}
$$

The same argument as before shows that if $f \neq 0$ in (34) then $f$ has a pole at zero and we set $f=F^{-1}, F(0)=0$. Setting $x=\alpha+\beta, y=\alpha$ and multiplying by $F(x) F(y)$ we transform (34) to

$$
\frac{F(y)-F(x)}{F(x-y)}=\frac{F(y)}{F(-x-y)}+\frac{F(x) F(y)}{F(-y) F(x+y)} .
$$

Differentiating (36) with respect to $x$ and setting $x=0$ we obtain

$$
-\frac{2 F^{\prime}(0)}{F(-y)}=\frac{2 F^{\prime}(-y) F(y)}{F^{2}(-y)} \text {. }
$$

As before, $F^{\prime}(0)=0$ leads to $F=0$ which is impossible, hence $F^{\prime}(0) \neq 0$ and normalizing $F^{\prime}(0)=1$ we get

$$
F^{\prime}(y) F(-y)+F(y)=0 .
$$

The same argument as in the previous case shows that $F$ is uniquely determined by $F^{\prime \prime}(0)=a$. A direct computation shows that $F(y)=y$ and $F(y)=a^{-1}\left(e^{a y}-1\right)$ satisfy not only (38) but also (36). Hence the BGG and the Demazure functions are the only solutions of (34). The same argument as in the $m=6$ case shows that (35) reduces to

$$
g(\beta) g(-\beta)-g(2 \alpha+\beta) g(-2 \alpha-\beta)=f(\beta) f(-\beta)-f(2 \alpha+\beta) f(-2 \alpha-\beta)
$$

which, as before, implies (32) and (33). Thus, assuming $f \neq 0, g \neq 0$, formulas (10) and (11) give the only solutions of the braid relations in the $m=4$ case.

The case $m=3$ is done in [7]. We only write the equations here:

$$
f(\alpha) f(\beta)+f(\alpha+\beta) f(-\alpha)=f(\alpha+\beta) f(\beta)
$$

and

$$
f^{2}(\alpha) f(\beta)+f(\alpha+\beta) g(\alpha) g(-\alpha)=f(\alpha) f^{2}(\beta)+f(\alpha+\beta) g(\beta) g(-\beta)
$$

and leave it to the reader to check that the previous argument applies once again. The theorem is proved.

REMARK. The "trivial" solutions with $f=0$ or $g=0$ are easy to find. For instance, if $f=0$ then all the equations $E_{2}, \ldots, E_{m}$ become trivial, hence $g$ is arbitrary. 
3. Applications. Theorem 1 gives the general form of representations of braid relations. The reader can easily find which values of the parameters $(a, b, c, h)$ correspond to the operators $B_{i}, D_{i}, G_{i}$ and $L_{i}$ of the introduction. Actually, it is possible that different sets of parameters yield the same representation.

PROPOSITION 1. (i) Two sets of parameters $\left(b_{1}, c_{1}, h_{1}\right) \neq\left(b_{2}, c_{2}, h_{2}\right)$ give the same $B G G$ type representation of braid relations if and only if $b_{1}=b_{2}, c_{2}=-c_{1}$ and

$$
h_{2}=h_{1} \frac{c_{1} x+b_{1}}{c_{1} x-b_{1}} \text {. }
$$

(ii) In the Demazure case different parameters $(a, b, c, h)$ yield different representations of braid relations.

We leave the proof of Proposition 1 to the reader. Recall that the Hecke algebra $H_{q}$ (see e.g. [15]) corresponding to a Coxeter group $W$ is the braid algebra $B$ with the additional relations

$$
\left(b_{i}-q\right)\left(b_{i}+1\right)=0, \quad i=1, \ldots, n .
$$

It is clear that $H_{q}=H_{1 / q}$ and that $H_{1}$ is the group algebra of $W$. The nil Hecke algebra $R[\mathbf{1 4}]$ is obtained from the braid algebra $B$ by adding relations

$$
b_{i}^{2}=0, \quad i=1, \ldots, n .
$$

THEOREM 2. (i) Let $T_{i}, i=1, \ldots, n$, be a nontrivial $B G G$ type representation of braid relations with parameters $(b, c, h)$. The algebra generated by the operators $T_{i}$ is isomorphic to $H_{1}$ for $c \neq 0$ and to $R$ if $c=0$.

(ii) Let $T_{i}, i=1, \ldots, n$, be a nontrivial Demazure type representation with parameters $(a, b, c, h)$. The algebra generated by $T_{i}$ is the Hecke algebra $H_{q}$ with $q=(b-c) / c$ if $c \neq 0$ and $H_{0}$ if $c=0$.

The proof is straightforward and we leave it to the reader.

It is often important to restrict the operators $T_{i}$ of a braid representation to natural subspaces of $M$. Such are the algebras $H, P$ and $Q$ of holomorphic, polynomial and rational functions on $\mathfrak{h}$. Slightly modifying Definition 2.2 of [9] we give the following.

DEFinition 2. Assume the previous notation and let $C_{i}, i=1, \ldots, n$, be a set of operators on $M$ preserving a natural subspace $\mathcal{F}$. We say that the operators $C_{i}$ form a calculus on $\mathcal{F}$ if for all $t \in \mathbf{C}$, any $w \in W$ and any decomposition $w=s_{1} \cdots s_{n}$ the operator

$$
C(w, t)=\left(s_{1}+t C_{1}\right) \cdots\left(s_{n}+t C_{n}\right)
$$

depends only on $w$ (not on the decomposition) and for any $t$ the correspondence $w \rightarrow C(w, t)$ is a representation of $W$.

THEOREM 3. Let $A$ be a generalized Cartan matrix with a nontrivial braid algebra $B$ and let $f \neq 0, g \neq 0$ be a pair of meromorphic functions. The operators

$$
C_{i}=f\left(\alpha_{i}\right)+g\left(\alpha_{i}\right) s_{i}, \quad i=1, \ldots, n,
$$

form a calculus (on $M$ ) if and only if

$$
C_{i}=b\left(1 / \alpha_{i}-\left(1 / \alpha_{i}\right) s_{i}\right)
$$


or

$$
C_{i}=b\left(1 / \alpha_{i}+\left(1 / \alpha_{i}\right) s_{i}\right)
$$

Equation (46) gives the only calculus on $P$ (the so-called BGG calculus [9]).

ProOF. If operators $C_{i}, i=1, \ldots, n$, form a calculus, then, by expanding (44) in powers of $t$, we see that $C_{i}$ satisfy the braid relations, thus Theorem 1 applies. Equation $1=\left(s_{i}+t C_{i}\right)^{2}=s_{i}^{2}+t\left(s_{i} C_{i}+C_{i} s_{i}\right)+t^{2} C_{i}^{2}$ shows that $C_{i}^{2}=0$, hence, by Theorem $2,(f, g)$ belongs to the BGG type with parameters $(b, 0, h)$. Normalizing $b=1$ we have $s_{i}+t C_{i}=t / \alpha_{i}+h\left(\alpha_{i}\right)\left(h\left(\alpha_{i}\right)^{-1}+t / \alpha_{i}\right) s_{i}$ which, by (10), gives $h=$ const and, in virtue of $(12), h= \pm 1$. If $h=-1$ the operators $C_{i}=\alpha_{i}^{-1}\left(1-s_{i}\right)$ preserve the space $P$, and if $h=1$ the operators $C_{i}=\alpha_{i}^{-1}\left(1+s_{i}\right)$ do not preserve $P$. Using Theorem 1 , it is straightforward to check that in both cases $C_{i}$ form a calculus (for $h=1$ it follows from [9]).

Theorem 3 answers a question of J. Bernstein [2]. In the course of its proof we have also proved the following.

COROLLARY 1. Let $W$ be a Weyl group with a nontrivial braid algebra, let $f, g \neq 0$ be meromorphic functions and define the operators $T_{i}$ by $(7), i=1, \ldots, n$. Then $s_{i} \rightarrow T_{i}$ is a representation of $W$ if and only if either

(i) $B G G$ case: $f$ and $g$ are given by (10) with $c= \pm 1, b$ and $h$ arbitrary; or

(ii) Demazure case: $f$ and $g$ are given by (11) with $b=0, c= \pm 1, h$ and $a \neq 0$ arbitrary.

\section{REFERENCES}

1. A. Arabia, Cohomologie T-equivariante de $G / B$ pour un groupe $G$ de Kac-Moody, C. R. Acad. Sci. Paris 302 (1986), 631-634.

2. J. Bernstein, Letter, 1986.

3. J. N. Bernstein, I. M. Gelfand and S. I. Gelfand, Schubert cells and the cohomology of spaces $G / P$, Uspekhi Mat. Nauk 28 (1973), 1-26.

4. N. Bourbaki, Groupes et algèbres de Lie, Chapitres 4, 5 et 6, Hermann, Paris, 1968.

5. M. Demazure, Invariants symetriques entiers des groupes de Weyl et torsion, Invent. Math. 21 (1973), 287-301.

6. __ Désingularization des variétés de Schubert generalizées, Ann. Sci. Ecole Norm. Sup. 7 (1974), 53-88.

7. S. Evens and P. Bressler, On certain Hecke rings, Proc. Nat. Acad. Sci. U.S.A. 84 (1987), 624-625.

8. E. Gutkin, Integrable systems with delta-potential, Duke Math. J. 49 (1982), 1-21.

9. __ Operator calculi associated with reflection groups, Duke Math. J. 55 (1987), 1-18.

10. _ Schubert calculus on flag varieties of Kac-Moody groups, Algebras, Groups and Geom. 3 (1986), 27-59.

11. E. Gutkin and P. Slodowy, Cohomologie des variétés de drapeaux infinies, C. R. Acad. Sci. Paris 296 (1983), 625-627.

12. V. Kac, Infinite dimensional Lie algebras, Progress in Math., Vol. 44, Birkhäuser, Boston, Mass., 1983.

13. V. Kac and D. Peterson, Cohomology of infinite dimensional groups and their flag varieties (to appear).

14. B. Kostant and S. Kumar, The nil Hecke ring and cohomology of G/P for a Kac-Moody group $G$, Adv. in Math. 62 (1986), 187-237.

15. G. Luszting, Equivariant $K$-theory and representations of Hecke algebras, Proc. Amer. Math. Soc. 94 (1985), 337-342.

Department of Mathematics, University of Southern California, Los ANGELES, CALIFORNIA 90089-1113 